\title{
The Effect of the Earth's and Stray Magnetic Fields on Mobile Mass Spectrometer Systems
}

\author{
Ryan J. Bell, ${ }^{1}$ Nicholas G. Davey, ${ }^{1,2}$ Morten Martinsen, ${ }^{3}$ R. Timothy Short, ${ }^{4}$ Chris G. Gill,, ${ }^{1,2}$ \\ Erik T. Krogh ${ }^{1,2}$ \\ ${ }^{1}$ Chemistry Department, Applied Environmental Research Laboratories (AERL), Vancouver Island University, Nanaimo, BC, \\ Canada \\ ${ }^{2}$ Chemistry Department, University of Victoria, Victoria, BC, Canada \\ ${ }^{3}$ Chemistry Department, Norwegian University of Science and Technology (NTNU), Trondheim, Norway \\ ${ }^{4}$ SRI International, St. Petersburg, FL, USA
}

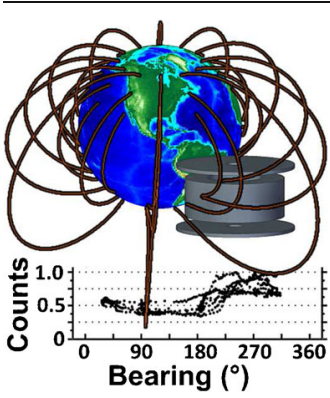

were paralleled by experiments performed with a mass spectrometer mounted on a rotating platform. Additionally, magnetically permeable metals were used to shield (1) the entire instrument from the Earth's magnetic field, and (2) the electron beam from both the Earth's and instrument's magnetic fields. Both simulation and experimental results suggest the predominant influence on directionally dependent signal variability is the result of the summation of two magnetic vectors. As such, the most effective method for reducing this effect is the shielding of the electron beam from both magnetic vectors, thus improving electron beam alignment and removing any directional dependency. The improved ionizing electron beam alignment also allows for significant improvements in overall instrument sensitivity. Key words: Mobile mass spectrometry, Membrane introduction mass spectrometry (MIMS), Online in situ analysis, Field portable, Earth's magnetic field, SIMION, Simulation, Mu-metal, Metglas, Electron beam trajectory deflection, Electron ionization

Received: 8 August 2014/Revised: 10 October 2014/Accepted: 11 October 2014/Published Online: 20 December 2014

\section{Introduction}

$\mathrm{T}$ he popularity of field portable mass spectrometry is growing as many of the technical hurdles of bringing laboratory equipment into the field are overcome [1-3]. On-site analysis

Electronic supplementary material The online version of this article (doi:10.1007/s13361-014-1027-4) contains supplementary material, which is available to authorized users.

Correspondence to: Ryan J. Bell; e-mail: ryan.bell@viu.ca; Chris G. Gill; e-mail: chris.gill@viu.ca requires that instrumentation can be transported conveniently, powered remotely, be free of self-contamination, and can be serviced in adverse conditions. Additionally, the instrumentation should be capable of data collection while moving, and overcome any additional hurdles resulting from changing environmental and sample conditions. For example, non-steady state or transient conditions are likely to be more prevalent and may require correction [4, 5]. Additionally, varied sample conditions, such as temperature, pressure, and humidity, may alter the relative instrumental response [3, 6-8], and changing environmental conditions may adversely affect the instrument itself [9]. To our knowledge, apart from a report by Short et al. 
(2000) [9], the effect of magnetic fields, such as the Earth's, on portable mass spectrometer signal intensity has not yet been addressed by the mass spectrometry community.

Electron beams that have a short path length or have sufficiently high energies are not meaningfully affected by weak magnetic fields. Additionally, weak magnetic fields are likely to have a negligible impact on the deflections of ion beams because of their significantly greater relative momentum. However, when precise, low-energy electron beams are employed, care must be taken to reduce the influence of uncontrolled magnetic fields. For example, photoelectron spectrometer components are intentionally lined with or constructed from magnetically permeable materials to limit the influence of magnetic fields [10]. Nickel-iron alloys such as mu-metal and other magnetically permeable materials have a magnetic permeability many times that of free space, and tend to absorb passing magnetic fields by providing a preferred location for field transmittance. Thus, when instrumentation is encased in mu-metal, magnetic fields inside the instrument are greatly reduced, avoiding unintended electron beam deflections. Although impractical in the context of portable mass spectrometry instrumentation, Helmholtz cages have also been employed to compensate for the effects of the Earth's magnetic field in testing facilities [11] and in high resolution cathode ray tubes [12].

In mass spectrometry, relatively low energy electron beams (e.g., $70 \mathrm{eV}$ ) are used in electron ionization (EI) sources. Though generally the ion source is located as close as possible to the mass analyzer, maintaining a flexible design sometimes necessitates transmission distances of several centimeters before electrons enter a small ion source electron aperture (e.g., < $3 \mathrm{~mm}$ diameter). In these cases, ionization efficiency (and therefore the instrument's relative sensitivity) will be dependent on the electron beam flux stability. Normally, in a stationary mass spectrometer, local magnetic fields are constant, and electron beam deflections go unobserved, or are compensated for by instrumental tuning optimizations. Occasionally, these fields are not constant; for example, we have observed that locating a solenoid pump or solenoid valve near the mass spectrometer's vacuum housing may result in a detectable effect on the signal intensity. For example, when sampling underwater vents in Yellowstone Lake in September 2003 with a portable underwater membrane introduction mass spectrometry system, an observable beat in the measured signals resulted from the operation of the solenoid sampling pump (Supplementary Figure S1). Post-deployment, it was discovered that the beat intensity was proportional to the proximity of the solenoid pump to the instrument's vacuum chamber. Encasing the pump in magnetically permeable metal resolved the issue (data not shown).

Field portable instrumentation also experiences varied magnetic fields as a result of changing its orientation with respect to the Earth's magnetic field. The resulting transient deflection of electron trajectory may generate a significant source of instrumental variability. In addition, the magnetic fields created by various mass spectrometer systems (e.g., vacuum pumps, inductors, or filament current) may also affect electron beam deflection. The summation of multiple magnetic field vectors will have an additive effect that is directionally dependent.

These effects are difficult to observe when instrumentation is operated in a GC mode because of the highly variable nature of the instrumental signal during a GC scan. Further, the effects are compensated for if instrument orientation is not changed between calibration and sample analysis, as recommended by instrument manufacturers. However, when operating in a continuous measurement mode, signal variability becomes easily observed when underway. In our case, the effect was highly intensified during operation with a sub-optimum electron lens voltage.

In this study, we characterize the influence of magnetic bearing on the relative instrument response of several field portable mass spectrometers, simulate the electron beam transmission efficiencies for several instrumental conditions, and demonstrate simple instrumental modifications that provide magnetic shielding, resulting in better signal stability and sensitivity gains.

\section{Experimental}

As noted above, we have experimentally observed a directional dependence of the relative instrument response for a variety of field portable mass spectrometers. To better understand this phenomenon, simulations were performed using SIMION (v8.1, Scientific Instrument Services Inc., Ringoes, NJ, USA) software that incorporated the influence of the Earth's magnetic field on an electron impact ion source as an instrument is rotated through $360^{\circ}$. Results from the rotation of a vertical electron beam were compared with that for a horizontal electron beam. Additional simulations explored the result of asymmetries generated from the use of a linear filament geometry as an electron source in an otherwise cylindrical system, and also the additive effect of local instrumental magnetic fields with the Earth's magnetic field. Simulation results were directly compared with experimental data collected in the laboratory.

To reduce the influence of magnetic fields on the relative instrumental response, three methods were explored experimentally and compared with simulations. First, the ion source's electron beam focus was altered, allowing the comparison of unfocused and well-focused electron beams. Next, the entire instrument was protected from the Earth's magnetic field by a mu-metal casing, reducing the presence of directionally dependent magnetic fields. Last, Metglas (an amorphous metallic ribbon material with very high magnetic permeability) was used to line the inside the instrument's vacuum chamber surrounding the electron beam to reduce the influence of both the Earth's and the much of the instrument's magnetic field. 


\section{Mobile Mass Spectrometer Relative Response Versus Orientation}

To demonstrate the effects of the orientation of the Earth's magnetic field on the relative instrumental response of different mobile mass spectrometers, experimental field data obtained from four different instruments were compared by analyzing a steady-state internal standard signal while the mass spectrometer's magnetic orientation (azimuth) was changed. All mass spectrometers utilized membrane sampling inlets employing polydimethylsiloxane capillary membranes maintained at constant temperatures. Membrane introduction mass spectrometry (MIMS) provides a direct analysis approach for the continuous quantitative online monitoring of specific analytes and internal standards. For additional background on MIMS, which is often employed in mobile mass spectrometry systems because of its simplicity, several reviews of the technique have been published [13-15].

The first instrument used was a field operable MIMS system made from a bench top, hyperbolic electrode quadrupole ion trap (QIT) (Model Saturn 2000; Agilent Technologies, Santa Clara, CA, USA), employing internal ionization. In this instrument, the electrons ejected from a hot filament are accelerated to $70 \mathrm{eV}$ and transmitted $\sim 1.7 \mathrm{~cm}$ before a focusing gate electrode guides the electrons through a $1 \mathrm{~mm}$ aperture in one of the ion trap's endcaps. The quantity of ions formed (and thus the relative signal intensity) is proportional to the number of electrons that pass through the $1 \mathrm{~mm}$ aperture. In addition, this instrument has the electron beam on a horizontal axis, parallel to the Earth's surface. To compare instrument response to magnetic orientation, a continuously infused $1 \mathrm{ppb}$ aqueous dimethylsulfide solution was analyzed using full scan mass spectra $(\mathrm{m} / \mathrm{z} 62)$ while operated onboard a moving marine research vessel (R/V Suncoaster, St Petersburg, FL, 1999) that was intentionally rotated $360^{\circ}$. Field orientation was determined by measuring the magnetic field perpendicular to the electron beam using a three-axis magnetometer (Model 2300; Honeywell, Morristown, NJ, USA) [9].

Data were also collected from a field portable cylindrical electrode quadrupole ion trap (CIT) (Model Griffin 400; FLIR Systems, West Lafayette, IN, USA), equipped with a custombuilt membrane interface. This instrument uses a $75 \mathrm{eV}$ electron beam for ionization within the ion storage volume of the trap. The electron path length from the filament to the end cap aperture is approximately $4.6 \mathrm{~cm}$ and a simple, three electrode cylindrical Einzel lens is used to focus the electron beam through a $2.5 \mathrm{~mm}$ aperture in one of the trap endcap plates. In this system, the electron beam axis is perpendicular to the Earth's surface (i.e., a vertical electron beam). Toluene- $d_{8}$ (used as an internal standard) was supplied to the membrane interface in air samples using a permeation tube (Kin-Tek, La Marque, TX, USA) housed in a thermally regulated permeation chamber in line with the membrane interface. The full scan instrument response with respect to orientation was obtained by operating the mass spectrometer inside a moving vehicle while analyzing exterior air continuously infused with $75 \mathrm{ppbv}$ toluene- $d_{8}(\mathrm{~m} / \mathrm{z}$ 98). Orientation was derived from GPS data while the vehicle was traveling in a 'figure eight' pattern.

The third instrument included in the study was a compact linear quadrupole mass spectrometer (Model Transpector CPM; Inficon, Syracuse, NY, USA) implemented as the mass analyzer in an underwater membrane introduction mass spectrometry system [16]. This mass spectrometer uses a closed electron ionization source, whereby $70 \mathrm{eV}$ electrons produced by the filament travel about $2 \mathrm{~mm}$ before passing through a 5 $\mathrm{mm} \times 2 \mathrm{~mm}$ slot. The data used here, collected from field studies made in Lake Maggoire, St Petersburg, FL, were also used in work published by Wenner et al. (2004) [17]. In this work, orientation was also derived from GPS data while the vehicle was travelling in a 'lawn mower' pattern. Electron beam direction (vertical vs. horizontal) was not determined.

The final instrument examined in this study was also a hyperbolic electrode quadrupole ion trap that utilizes an external electron ionization source (Model GCQ; Finnigan MAT, San Jose, CA, USA). This system employs an ion source in which $70 \mathrm{eV}$ electrons from the filament are collimated into a spiraling beam by two permanent magnets $(\sim 300-400 \mathrm{G})$. The focused electrons are passed through two apertures (each about $2.6 \mathrm{~mm}$ diameter) over $4.8 \mathrm{~mm}$ distance prior to entering the ion source, travelling on a vertical axis (i.e., perpendicular to the Earth's surface). Ions generated in the source are subsequently injected into the ion trap by a compact ion lens system. Full scan data $\left(\mathrm{m} / \mathrm{z}\right.$ 98) from a toluene- $d_{8}$ internal standard infused into ambient air at $341 \mathrm{ppbv}$ were obtained in the same manner as described for the CIT (vide supra). The data used here, collected while conducting field measurements in Tacoma, WA, was analyzed as part of a study published by Davey et al. (2014) [1]. Orientation was derived from GPS data.

\section{Electron Beam Deflection Simulations}

Ten simulations conducted using SIMION software are presented to understand the contributing factors and possible solutions to unintended electron beam deflections by magnetic fields in a mass spectrometer ion source. The electron beam was simulated as it traveled from the electrons' origin, through the focusing lens, and either passing through the end plate aperture or impacting the endplate. For these simulation studies, the ion source geometry and voltages of the CIT (vide supra) were used. The 2D-cylindrical model used to simulate the CIT ion source and transmission efficiency is shown in Figure 1. Constant values for the simulations included the electron deflector plate $(-10 \mathrm{~V})$, the ion trap endplates $(0 \mathrm{~V})$, and ring electrode $(75 \mathrm{~V})$, the initial electron kinetic energy $(0.2 \mathrm{eV})$, mean electron source location $(\mathrm{x}=0 \mathrm{~mm}, \mathrm{y}=0 \mathrm{~mm})$, and filled electron axial cone angle $\left(80^{\circ}\right)$. The initial electron kinetic energy was calculated assuming a $2050^{\circ} \mathrm{C}$ filament [18], using a Maxwellian distribution [19, 20]. Simulations were performed using 10,000 electrons at each of eight rotational steps $\left(45^{\circ}\right)$ relative to the Earth's magnetic field, with an additional ninth condition of no magnetic field from the Earth. Set-points that 


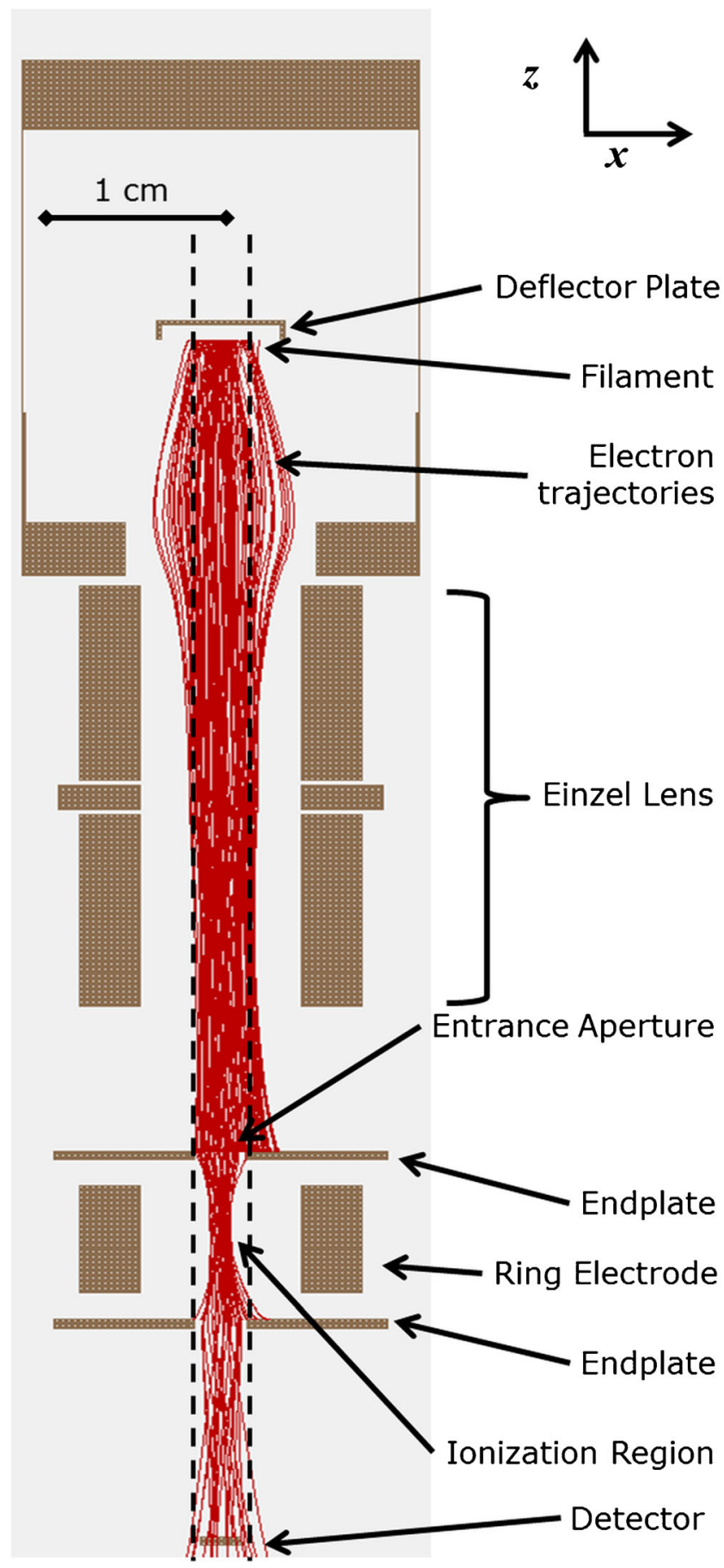

Figure 1. SIMION model approximating the CIT ion source shown in a vertical orientation relative to the Earth's surface ( $x y$ plane). The electron trajectories shown in this example are influenced the Earth's magnetic field, the sum of which is out of the page $(y)$ and, as a result, electron deflection is rightward $(x)$. The dashed reference lines demarcate the entrance aperture's $x$ position and are intended to help visualize electron deflection

were varied between simulations are summarized in Table 1. Intensities for the Earth's magnetic field in Nanaimo, Canada $\left(B_{x}=0.18 \mathrm{G}, B_{y}=0.054 \mathrm{G}\right.$, and $\left.B_{z}=0.51 \mathrm{G}\right)$ were obtained using the IGRF function from the Matlab (Mathworks, Natick,
MA, USA) file exchange, and confirmed using the an Android smartphone (Model Incredible; HTC Corporation, Taoyuan City, Taiwan). The smartphone contained a vector magnetometer that was monitored and logged using the AndroSensor (ver. 1.9.4.4a) application.

Simulations 1 through 3 were conducted to test whether the cause of observed signal variations in field measurements could be due to the relative orientation of the Earth's magnetic field. The electron beam in these simulations was oriented perpendicular to the Earth's magnetic field (i.e., a vertical electron beam), as is the case for the normal operation of the CIT system. Simulation 1 (intended to be the idealized case) was conducted with all electrons originating from a point source. Simulation 2 was identical, except that the origin of the electrons approximated the linear filament found in the CIT system. Here, the electrons were sourced from a Gaussian distribution $(\mathrm{SD}=0.3 \mathrm{~mm}$ ) along a line in the $y$ direction. Simulation 3 was performed using the same conditions as Simulation 2, with the exception of an additional secondary magnetic field. This secondary field was not rotated with the Earth's magnetic field, thus simulating the measured magnetic field originating within the instrument itself. The intensity of this vector $(B x=-0.047 \mathrm{G}, B y=0.21 \mathrm{G}$, and $B z=-0.63 \mathrm{G})$ was determined using a magnetometer adjacent to the instrument's vacuum housing, and subtracting the Earth's magnetic contributions. Because the magnetic field in the instrument housing varies strongly within a few centimeters, this determination is likely one of the largest sources of error for these simulations. Simulations 4 through 6 were parallel experiments to Simulations 1 through 3 except that the magnetic vectors were altered to reproduce the Earth's field rotating about the instrument's $y z$ plane, simulating a horizontal electron beam. The horizontal beam has a distinctly different symmetry with respect to the Earth's magnetic field than the vertical electron beam. Although the CIT was not designed to be operated in this orientation, a horizontal electron beam was employed in the hyperbolic electrode quadrupole ion trap (i.e., Saturn 2000, vide supra).

\section{Electron Beam Defocusing Studies}

Together, Simulations 3, 7, and 8 were designed to determine the importance of electron trajectory deflection when the electron beam is refocused by altering the Einzel lens voltage while in the vertical electron beam configuration. Simulation 3 was used as a reference, employing the manufacturer recommended set-point of $25 \mathrm{~V}$ for the Einzel lens, whereas Simulation 7 and 8 used a lens voltage of $75 \mathrm{~V}$ and $200 \mathrm{~V}$, respectively.

For direct comparisons with the simulations, analogous experiments were conducted on the CIT system. Toluene was used as a constantly infused analyte (via $70 \mathrm{ppb}_{\mathrm{v}}$ air samples continuously infused to the membrane interface), and the instrument was located on a free moving turntable where $0^{\circ}$ is magnetic north in Nanaimo, Canada. In this case, the instrument analyzed the toluene using an MS/MS scan $(m / z 91 \rightarrow 65$, $10 \mu \mathrm{A}$ electron current, $150 \mathrm{~ms}$ ionization time, $1.5 \mathrm{~V}$ dissociation voltage, and $20 \mathrm{~ms}$ dissociation time). 
Table 1. Parameters Varied Between Simulations of the CIT Ion Source. Electron Beam Orientation is with Respect to the Earth's Surface (i.e., the $x y$ plane). Lens and Ion Trap Design are Depicted in Figure 1 and Described in Text

\begin{tabular}{|c|c|c|c|c|c|c|c|c|c|c|}
\hline \multirow[t]{2}{*}{ Sim. \# } & \multirow[t]{2}{*}{ Description } & \multirow[t]{2}{*}{$\begin{array}{l}\text { Electron beam } \\
\text { orientation }\end{array}$} & \multirow[t]{2}{*}{ Lens (V) } & \multirow{2}{*}{$\begin{array}{l}\text { SF of electron } \\
\text { source on y } \\
(\mathrm{mm})\end{array}$} & \multicolumn{3}{|c|}{ Earth’s field (G) } & \multicolumn{3}{|c|}{$\begin{array}{l}\text { Instrument } \\
\text { referenced field (G) }\end{array}$} \\
\hline & & & & & $B x$ & By & $B z$ & $B x$ & By & $B z$ \\
\hline 1 & Earth's field only & vertical & 25 & 0 & 0.18 & 0.054 & 0.51 & 0 & 0 & 0 \\
\hline 2 & Earth's field + filament & vertical & 25 & 0.3 & 0.18 & 0.054 & 0.51 & 0 & 0 & 0 \\
\hline 3 & Earth's field + filament + instrument's field & vertical & 25 & 0.3 & 0.18 & 0.054 & 0.51 & 0.21 & -0.047 & -0.63 \\
\hline 4 & Earth's field only & horizontal & 25 & 0 & 0.18 & 0.054 & 0.51 & 0 & 0 & 0 \\
\hline 5 & Earth's field + filament & horizontal & 25 & 0.3 & 0.18 & 0.054 & 0.51 & 0 & 0 & 0 \\
\hline 6 & Earth's field + filament + instrument's field & horizontal & 25 & 0.3 & 0.18 & 0.054 & 0.51 & 0.21 & -0.047 & -0.63 \\
\hline 7 & $\begin{array}{l}\text { Earth's field + filament + instrument's } \\
\text { field + altered focus }\end{array}$ & vertical & 75 & 0.3 & 0.18 & 0.054 & 0.51 & 0.21 & -0.047 & -0.63 \\
\hline 8 & $\begin{array}{l}\text { Earth's field + filament + instrument's } \\
\text { field + very altered focus }\end{array}$ & vertical & 200 & 0.3 & 0.18 & 0.054 & 0.51 & 0.21 & -0.047 & -0.63 \\
\hline 9 & $\begin{array}{l}\text { Earth's field + filament }+ \text { instrument's } \\
\text { field }+ \text { mu-metal around instrument }\end{array}$ & vertical & 25 & 0.3 & 0.060 & 0.018 & 0.17 & 0.21 & -0.047 & -0.63 \\
\hline 10 & $\begin{array}{l}\text { Earth's field + filament }+ \text { instrument's } \\
\text { field + Metglas in vacuum chamber }\end{array}$ & vertical & 25 & 0.3 & 0 & 0 & 0.51 & 0 & 0 & -0.63 \\
\hline
\end{tabular}

\section{Magnetic Field Shielding Effects}

Simulations 3, 9, 10, and their associated parallel experiments (again, using the CIT) were performed to determine the effectiveness of using magnetically permeable materials to reduce the intensity of the Earth's magnetic field experienced by the mass spectrometer. Simulation 3 was used as a reference point, and Simulation 9 was designed to match the conditions that were determined after paneling the entire instrument with mumetal. In this case, the instrument's outside casing was covered with about $1.6 \mathrm{~m}^{2}$ of $0.1 \mathrm{~mm}$ thick nickel alloy foil, or mumetal $(\mu \approx 50,000)$ (Product Number 8912 K32; McMaster Carr, Elmhurst, IL, USA), held in place with adhesive tape. Magnetic fields inside the instrument after the mu-metal was installed were determined by locating the magnetometer next to the vacuum housing and rotating the operational instrument through $360^{\circ}$. The magnitude of instrument's and Earth's magnetic field vectors were resolved using custom Matlab code. It was determined that the mu-metal resulted in a $66 \%$ reduction in the intensity of the Earth's $x, y$, and $z$ magnetic field vectors. The instrument's internal magnetic fields changed slightly, but this was highly dependent on the exact location of the magnetometer. Because of this, it was decided to retain the original numbers for the instrument's magnetic field for consistency with each simulation.

Simulation 10 was designed to match the conditions that were determined after the interior of the vacuum chamber was lined with a 10 layer thick cylinder of $0.015 \mathrm{~mm}$ thick Metglas ( $\mu \approx 290,000$, Metglas 2075 M; Metglas, Conway, SC, USA) mounted on the interior wall of the CIT instrument's vacuum chamber. Several small holes were cut in the Metglas cylinder to allow the necessary electrical feed-through connections required for normal operation. The magnetic fields in the vacuum housing were characterized by locating the magnetometer within the vacuum chamber and rotating the (nonoperational) instrument through $360^{\circ}$. It was determined that the $x y$ components of both the Earth and instrument magnetic fields were negligible. Because the top and bottom of the chamber were not protected by Metglas, there was a significant $z$ field. Over the course of the study, it was also determined that the difference between the instrument's internal magnetic field was small when operating or not, suggesting the instrument's fields are primarily sourced from the permanent magnets in the turbomolecular vacuum pump (Model TC100; Pfeiffer Vacuum, Asslar, Germany). Neither the mu-metal foil nor the Metglas were annealed for this work, which would further increase their relative magnetic permeability.

Figures were generated using Matlab, and the data plotted with respect to the field orientation was best fit with a line determined by a nonlinear fit to $y=a+b * \sin (c+x)+$ $d * \sin (2 *(e+x)$. Error bars shown in simulated data represent the SD of electron transmission through the aperture for the 10,000 simulated electrons binned into groups of 10. Error bars shown in experimental data represent the SD of the 24 steady state signal measurements.

\section{Results}

\section{Mobile Mass Spectrometer Sensitivity Versus Directional Bearing}

For the purpose of demonstrating the in-field response of a mobile mass spectrometer to relative to the Earth's magnetic orientation, internal standard signal intensities for the four mobile mass spectrometer systems examined are plotted in Figure 2. Data shown in Figure $2 \mathrm{a}$ and $\mathrm{b}$ were produced by quadrupole ion traps using internal ionization directly within the ion storage volume, requiring the injection of electrons through an endcap aperture. In both cases, there was an observed dependence on directional bearing in which the signal intensity displays one maxima per $360^{\circ}$ rotation. Shown in Figure $2 \mathrm{c}$ are data obtained for a quadrupole ion trap with an external ion source that generates ions using electrons focused 


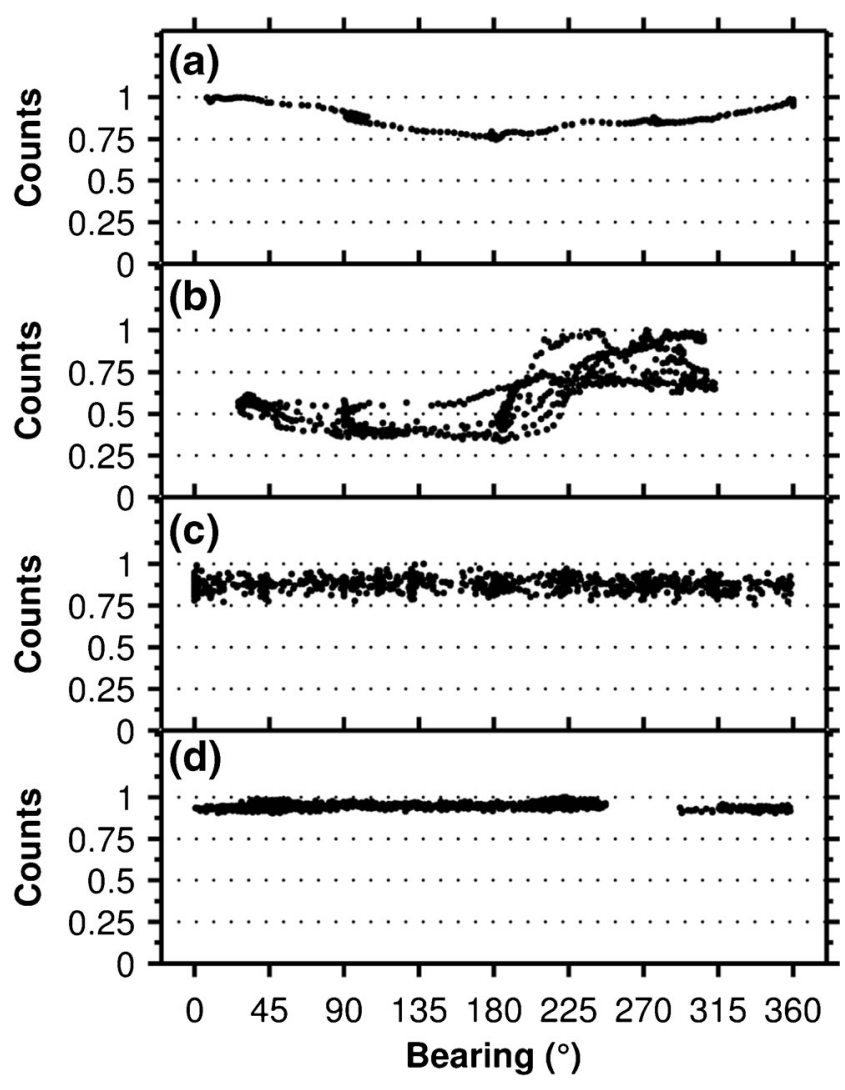

Figure 2. Moving mass spectrometers' response to an internal standard plotted versus directional bearing. (a) An ion trap with internal ionization (e.g., electrons are injected into the ion trap where ionization occurs) and an electron beam horzontal to the Earth's $x y$ plane. (b) Another ion trap with internal ionization but vertical electron beam. (c) An ion trap with external ionization (e.g., ionization occurs outside the trap and ions are subsequently injected into the trap). (d) A closed source quadrupole mass spectrometer. Models and ion source designs are further described in the Experimental section. It is notable that only instruments with internal ionization exhibit a directional dependency

by a strong magnetic field. Ions produced in this system were subsequently injected into the ion trap. The data illustrate the external ion source exhibited no signal dependence with respect to magnetic orientation, suggesting the strong magnets focusing the electrons into the ion source effectively overcome any influence from stray magnetic fields. Similarly, the data collected using the single quadrupole mass spectrometer, shown in Figure 2d, indicated no signal dependence with respect to the directional bearing. This mass spectrometer has a very short electron trajectory and a relatively large electron aperture slot in the ion source; as such, it is unlikely that electron beam deflections were significant under such conditions.

On the whole, the field data indicate mass spectrometers with relatively long electron trajectories and small entrance apertures (e.g., internal ionization ion trap type systems) are strongly affected by directionality, whereas short path lengths, large apertures, and strong collimating magnetic fields are likely to negate any directional bearing dependency. Regardless of horizontal or vertical ionizing electron beam axes relative to the earth's magnetic field, one maximum per rotation was observed in the directional dependence of the relative instrumental sensitivity of the mobile mass spectrometer systems exhibiting this effect.

\section{Ion Source Simulations}

Simulation 1 has an ion source that is cylindrically symmetric and has the electron beam traveling in the vertical direction. As a result, the transmission of electrons through the endplate aperture in Simulation 1, which rotates the Earth's magnetic field about the vertical axis, showed no reproducible directional dependence (Figure 3a). The mean transmission was $8.7 \%$, which is almost half the $14.7 \%$ that is simulated to occur without the Earth's field present. The inclusion of the Earth's field, while reducing electron transmission by deflecting the center of the electron beam away from the aperture, had no directional dependency attributable to the cylindrical symmetry and vertical orientation.

Simulation 2 adds an asymmetry to the system by including the length of the filament when simulating the origin of each electron. Set-points were such that over $99.7 \%$ of electrons were sourced from the middle $1 \mathrm{~mm}$ section of filament wire. Figure 3 a illustrates that there was little effect from this asymmetry. When the length of the filament was exaggerated (e.g., electrons sourced from a $5 \mathrm{~mm}$ long filament, data not shown) two maxima per rotation were observed. This would be the expected result from a system with bilateral symmetry. The inclusion of the asymmetry did not have a significant effect on the directional dependency, and the resulting two maxima were not observed in any of the experimental field data.

Simulation 3 is the closest representation of the operating conditions of the CIT instrument. In addition to including the accurate representation of the filament and the Earth's magnetic field, it includes a static secondary field that is referenced to the instrument itself. A strong directional dependency with one maximum per rotation is illustrated in Figure 3a. In this simulation, the Earth's magnetic field is of similar magnitude to the static field. Therefore, when the two fields interfere destructively (e.g., at $135^{\circ}-180^{\circ}$ ), the electrons were not significantly deflected and experienced a higher transmission efficiency through the aperture. However, when the two fields interfere constructively (e.g., at $315^{\circ}-360^{\circ}$ ), the electrons were strongly deflected, and fewer electrons were transmitted. Figure $4 \mathrm{a}$ depicts, in greyscale, the relative likelihood of an electron's position as it reaches the ion trap's endplate. The effect of the summation of the two magnetic field vectors becomes clear, demonstrating why the constructive and destructive interference of the two magnetic fields has the strongest influence on directional dependence. It is also worth noting that under 'standard conditions' (i.e., Simulation 3), while the electron beam was unfocused, there was still a greater electron density in the center of the beam. Whether or not the central area of the 

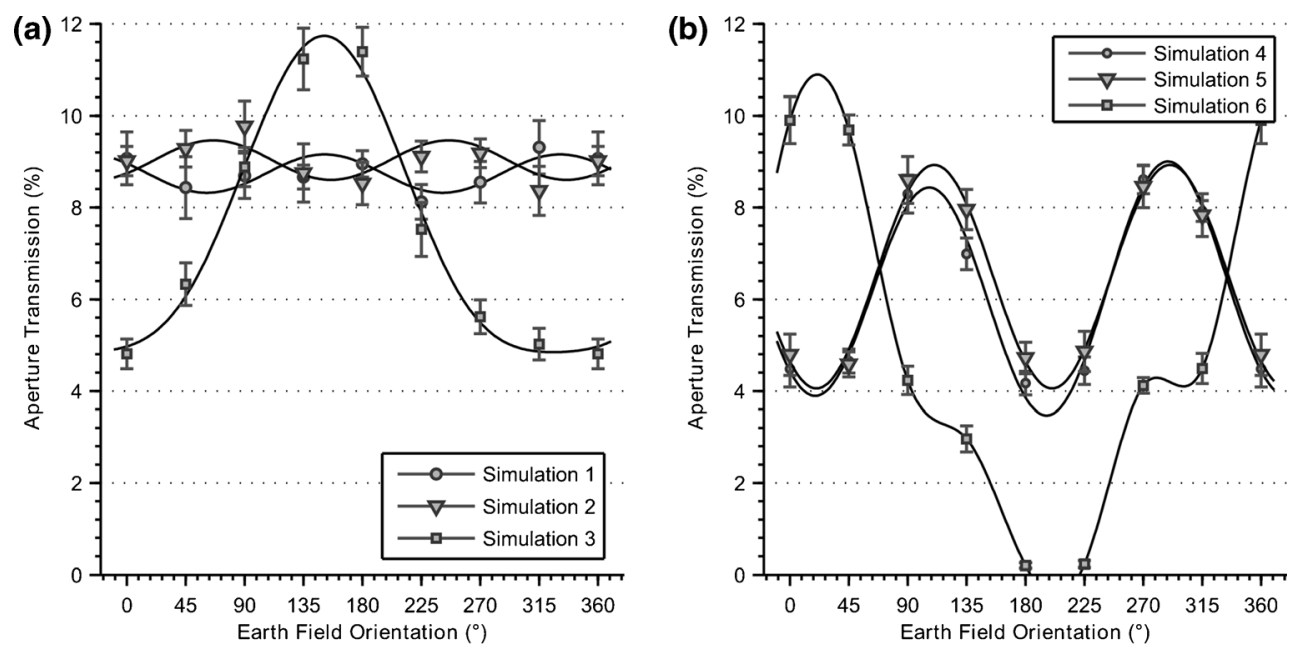

Figure 3. Simulation of electron transmission of a vertical (a) and a horizontal (b) electron beam through endplate aperture versus the Earth's field orientation. Simulation 1 and 4, Earth's field + point source filament; Simulation 2 and 5, Earth's field + transverse filament wire; and Simulation 3 and 6, Earth's field + transverse filament wire + instrument's field

electron beam passes the aperture had a significant effect on the number of electrons ultimately entering the ion trap and subsequently ionizing neutral molecules.

In the case of the vertical electron beam, Simulation 3 (Figure 3a) accurately predicted the one maximum per rotation signal observed in the field measurements for the mobile mass spectrometers. Given the additional asymmetry that results from a horizontal electron beam, one might expect two maxima in such a system. However, Figure 2a depicts a mass spectrometer in the field with a horizontal electron beam - also with one maximum per rotation. Simulation 4 (horizontal electron beam, point source electron source, Figure $3 b$ ) shows that the effect of the Earth's field alone does, in fact, create a two maxima directional dependency. The inclusion of the filament wire in the electron source has a rather small effect (Figure 3b, Simulation 5).
However, Simulation 6, which included the additional instrument referenced magnetic field, shows a dramatic one maximum directional dependency (Figure 3b). The constructive/ destructive interference of the two fields clearly had a very strong effect on the system and explains why the QIT, with a horizontal electron beam, displays a dominant one maximum direction dependency on the field. It is noteworthy that the dimensions of the QIT are different from the CIT and the magnitude of signal variation was not expected to be in agreement between Simulation 6 (Figure 3b) and Figure 2a.

\section{Reducing the Relative Response Variability}

Having established a plausible mechanism for the variation in signal intensity with respect to the instrument's magnetic

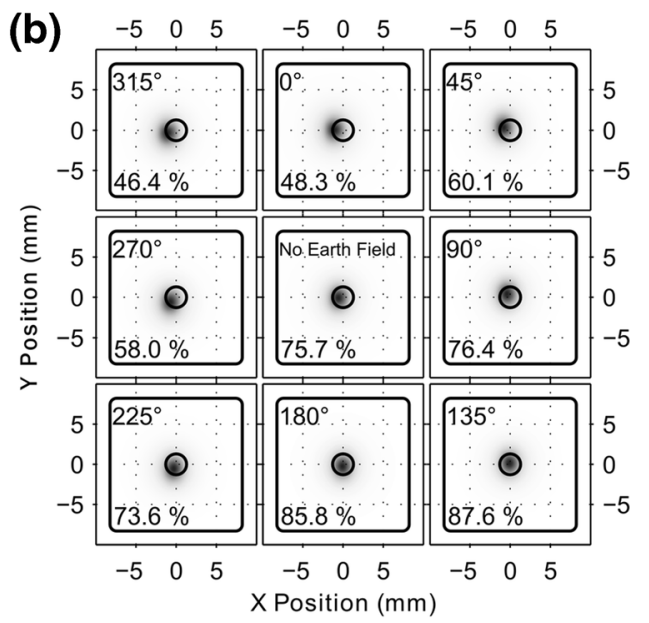

Figure 4. Density maps indicating the $x y$ location of electrons as they reach the ion trap endplate for Simulations $3[25 \mathrm{~V}$ lens, (a)] and 8 [200 V lens, (b)] under different magnetic bearing conditions. Each subplot is calculated from 10,000 electrons, the dark grey represents high probability of an electron at that position, and light grey represents low probability. The outside rectangle represents the outside edge of the endplate and the central circle represents the endplate aperture. Magnetic bearing and percent of electrons passing the aperture are indicated 
orientation, Simulation 3 was used as a starting point for subsequent simulations and experiments. These studies were aimed at determining an effective way to reduce the observed sensitivity dependency related to directional bearing. Simulations 3 and 7-10 were matched with a parallel experiment performed in the lab with a CIT instrument mounted on a turntable. Simulations 7 and 8 simulate the results when the electrons are not focused according to manufactures specifications by the Einzel lens prior to the aperture. Figure $4 \mathrm{~b}$ shows the improved aperture transmission relative to Figure $4 \mathrm{a}$ resulting from altering the lens voltage from $25 \mathrm{~V}$ (Simulation 3) to $200 \mathrm{~V}$ (Simulation 8). Experimental data are in relatively good agreement with Simulations 3,7 , and 8 (Figure 5). These data suggest that a defocused electron beam (Simulation 3) does not necessarily reduce the directional dependency, but does reduce instrumental sensitivity. Not shown are simulations with an increased secondary field. In these simulations, a well-focused beam can result in an extreme directional dependency as the entire beam may be transmitted or rejected by the aperture depending on the state of interference with the Earth's magnetic field.

Simulation 9 compared electron aperture clearance with Simulation 3 after a reduction in the Earth's magnetic field by adding mu-metal on the outside of the instrument, and Simulation 10 compared transmittance after a reduction in both the Earth's and instrument's fields by adding Metglas on the inside of the vacuum chamber. These simulations were directly compared with instrumental data collected under the same magnetic conditions (Figure 6). Simulation 3 indicates a $58.7 \%$ variation in signal intensity as a result of the additive effect of the Earth's and the instrument's fields. Simulation 9 indicates that this is reduced to a $21.7 \%$ signal variability with the addition of mu-metal on the outside of the instrument (a reduction of $62.9 \%$ in the signal variability). This result is comparable to the experimental data, which demonstrated a $47.4 \%$ reduction in actual signal variance between the standard and mu-metal conditions. Simulation 10 indicates we can expect $4.9 \%$ signal variability (a reduction of $93.2 \%$ in the signal variability) as a result of the Metglas addition. This result is comparable to the actual data from the CIT that displayed a 92.3\% reduction in signal variability between the standard and Metglas conditions. This reduction made the relative response variability due to the instrument's magnetic orientation virtually indistinguishable from other instrumental noise.

\section{Discussion}

Regardless of the specifics of the mass spectrometer instrument symmetry, the results of these studies suggest the best way to minimize directional response dependency is to shield low energy electron beams from both the Earth's and instrument's magnetic fields. In doing so, the electron beam can be properly aligned for optimum ionization, regardless of the orientation of the instrumentation during mobile measurements. Both simulation and experimental results indicate that in addition to significantly reducing the signal variability during deployment, the focusing lens may be tuned to ensure a higher electron throughput yielding significant improvements in sensitivity. Simulations indicate that a well-tuned lens can improve instrumental sensitivity nearly 10 -fold. However, it should be noted that shielding of the electron beam from stray magnetic fields becomes imperative in this case, as it is possible that the tightly focused electron beam could miss the entrance aperture entirely. With increased sensitivity, the opportunity to reduce the required filament emission current arises; this may be desirable

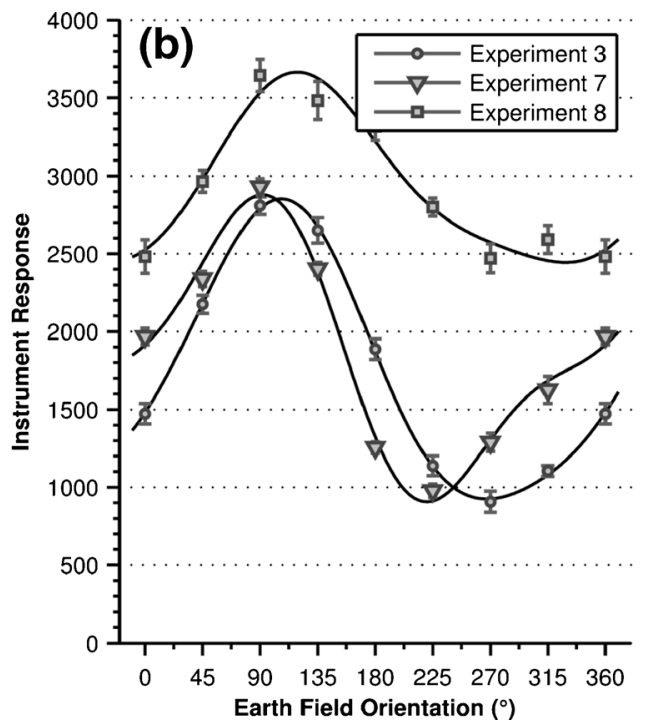

Figure 5. Electron transmission versus Earth's field orientation under varied lens set-points. (a) Illustration of the percentage of electrons transmitted with a lens set-point of $25 \mathrm{~V}$ (Simulation 3), $75 \mathrm{~V}$ (Simulation 7), and $200 \mathrm{~V}$ (Simulation 8); (b) plots the instrument response to an internal standard during a parallel experiment performed on a mass spectrometer under the same conditions as the simulation 

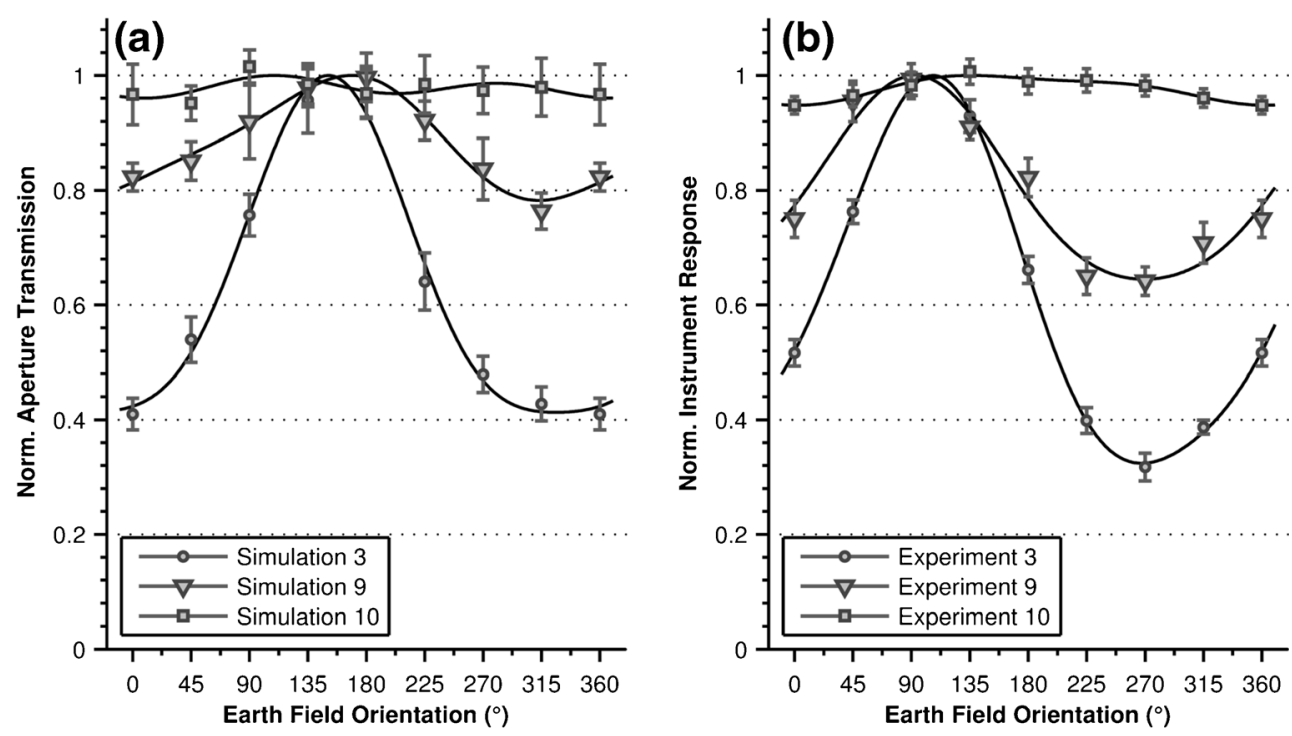

Figure 6. Electron transmission versus Earth's field orientation under varied magnetic field conditions. (a) The percentage of simulated electrons transmitted through the aperture with no protection from magnetic fields (Simulation 3), $66 \%$ reduction of the Earth's magnetic field by an exterior mu-metal casing (Simulation 9), and 95\% reduction of both the Earth's field and instrument's field by interior Metglas (Simulation 10); (b) plots the relative instrumental response to an internal standard during a parallel experiment performed on a CIT mass spectrometer operated under the same conditions as the simulation

when the vacuum chamber is under relatively oxidative conditions, as is common in MIMS systems. This would yield longer filament lifetimes and lower the signals from background gases $[7,21]$.

Another factor that should be considered is the electron current control feedback mechanism. In order to produce a constant electron ionization current, ion sources are designed to maintain a constant flow of electrons by using a feedback control system. Typically, there are two methods to monitor and control filament emission current. One is achieved by monitoring the filament emission current using the filament power supply circuitry; this methodology provides no correction for the variations in transmission through the ion trap entrance aperture. Another method is to monitor the electron current on an aperture plate in the ion optical system (e.g., the ion trap entrance aperture); use of this method assumes that the current collected on this plate is a constant fraction of the transmitted electron current. This methodology provides feedback control for emission control circuitry but unfortunately may serve to exaggerate the directional dependence. In this case, fewer electrons may be entering the ionization region because of deflection by external magnetic fields. With more electrons striking the aperture plate, this would provide erroneous feedback that acts to decrease filament current, resulting in further reduction of the transmitted electron current. Another feedback option, however, could be to measure the electron current on the ion trap exit endplate. This feedback mechanism would compensate for variations in transmitted electron current by increasing filament current when transmission is decreased. Since only the transmitted electrons contribute to the feedback, this method would compensate for deflections resulting from the Earth's magnetic field. However, it may prove impractical because of the radio frequency trapping fields of the trap, which may direct a portion of transmitted electrons into the ring electrode instead.

Additionally, the use of automatic gain control may help compensate for the observed phenomena, although gain control was fixed during these studies. Since mass spectrometers can have several scan types and pre-scans, and the directional dependence of the full scan and an MS/MS scan varies slightly with the CIT (data not shown). Such variability is likely due to changes in ionization time estimated by instrumental gain control pre-scans. Investigation of this phenomenon was beyond the scope of the current study.

Solutions not explored in this work include the use of magnetically permeable paints or magnetically permeable components, especially those components closest to the electron beam (e.g., the lenses and vacuum housing). Additionally, simulations suggest that in some situations where precision is key, it may be prudent to use a pointed filament that would give a more cylindrically symmetric electron beam than that produced by a linear filament wire perpendicular to the electron beam. Locally shielding specific instrumental components that are contributing to the instrument's magnetic field may also be beneficial, especially if it is not practical to sufficiently shield the electron beam.

Some instrumental components can have very strong magnetic fields (e.g., from the filament current, turbo pump magnetic bearings, or induction coils). Further, if the component generates cyclical fields (e.g., a motor or pulsing solenoid), the field coming in and out of phase with specific mass scans may significantly affect instrument stability. Depending on aliasing and frequency reproducibly, this noise may show up as a beat (as demonstrated in Supplementary Figure S1) or as random 
noise. A noteworthy source of instrumental magnetic fields may be the electric current through the filament. A filament operating at 5 amps (a high but not unusual current for an ion source), would have a field equal to the Earth's at a distance of $2 \mathrm{~cm}$. While this field intensity would decrease rapidly with distance (Ampere's Law), it will be very intense in close proximity to the filament. This will cause significant deflection near the filament and be very difficult to prevent; however, the subsequent focusing via the Einzel lens dampens the effect of deflections prior to and within the lens. Magnetic fields located between the lens and ion trap entrance endplate will have the most significant impact on electron beam deflections. This effect can be observed through careful examination of electron trajectories relative to the aperture reference lines shown in Figure 1, and suggests this effect could be minimized by placing the Einzel lens as close as possible to the ion trap endplate.

Last, as this study occurred in Nanaimo, Canada, all simulations were done assuming a field at that location (latitude, longitude, and near sea-level altitude). At higher latitudes, the Earth's magnetic $z$ vector is rather strong and subsequently $x$ and $y$ vectors are rather weak. Since the $z$ vector does not contribute to the deflection of electrons travelling in the vertical axis, the effects observed in this manuscript will be stronger at more equatorial latitudes.

\section{Conclusions}

To our knowledge, this is the first rigorous study of the influence of stray magnetic fields from the Earth and/or instrumental sources on the relative signal response and sensitivity of portable mass spectrometers. Signal variability is observed with instruments in which ionizing electrons have long path lengths and must pass through a small aperture, and are observed when the instrument is physically moved with respect to the Earth's magnetic field. It was found that the predominant source of signal variability is a result of the vector sum of the Earth's magnetic field and a secondary, instrument-referenced magnetic field. Removing either one will result in a significantly reduced directional dependence. Preferably, both fields can be shielded from the electron beam by the addition of magnetically permeable material to the vacuum chamber. In this case, directional dependence will become increasingly negligible as the deflection of the electron beam is eliminated. Subsequently, the optimum alignment of electron ionization beams may allow for improved electron beam focusing and significant gains in instrument sensitivity.

\section{Acknowledgments}

The authors gratefully acknowledge the assistance of the staff of the Center for Ocean Technology, College of Marine Science, and the C. D. Simpson Laboratories at the University of Washington for their assistance with the collection of various field data. The authors also thank the students and staff of the
Applied Environmental Research Laboratories (AERL) for their assistance with this project, and the notably insightful input from Kyle Duncan. They also thank two anonymous reviewers for their thoughtful reviews and intellectual contributions to this manuscript. They are grateful to Vancouver Island University and the University of Victoria for their ongoing support of graduate students and the Natural Science and Engineering Research Council of Canada for support.

\section{References}

1. Davey, N.G., Fitzpatrick, C.T.E., Etzkorn, M.J., Martinsen, M., Crampton, R.S., Onstad, G.D., Larson, T.V., Yost, M.G., Krogh, E.T., Gilroy, M., Himes, K.H, Saganić, E.T., Simpson, C.D., Gill, C.G.: Measurement of spatial and temporal variation in volatile hazardous air pollutants in Tacoma, WA using a mobile membrane introduction mass spectrometry (MIMS) system. J. Environ. Sci. Health A. 49, 1199-1208 (2014)

2. Taylor, S., Bierbaum, V.M.: Focus on harsh environment mass spectrometry. J. Am. Soc. Mass Spectrom. 19, 1375-1376 (2008)

3. Huang, G., Gao, L., Duncan, J., Harper, J.D., Sanders, N.L., Ouyang, Z., Cooks, G.R.: Direct detection of benzene, toluene, and ethylbenzene at trace levels in ambient air by atmospheric pressure chemical ionization using a handheld mass spectrometer. J. Am. Soc. Mass Spectrom. 21, 132-135 (2010)

4. Short, R.T., Toler, S.K., Kibelka, G.P.G., Rueda Roa, D.T., Bell, R.J., Byrne, R.H.: Detection and quantification of chemical plumes using a portable underwater membrane introduction mass spectrometer. TrAC Trends Anal. Chem. 25, 637-646 (2006)

5. Janfelt, C., Lauritsen, F.R., Toler, S.K., Bell, R.J., Short, R.T.: Method for quantification of chemicals in a pollution plume using a moving membranebased sensor exemplified by mass spectrometry. Anal. Chem. 79, 53365342 (2007)

6. Bell, R.J., Short, R.T., van Amerom, F.H.W., Byrne, R.H.: Calibration of an in situ membrane inlet mass spectrometer for measurements of dissolved gases and volatile organics in seawater. Environ. Sci. Technol. 41, 81238128 (2007)

7. Bell, R.J., Short, R.T., Byrne, R.H.: In situ determination of total dissolved inorganic carbon by underwater membrane introduction mass spectrometry. Limnol. Oceanogr. Methods 9, 164-175 (2011)

8. Diaz, J.A., Pieri, D., Arkin, C.R., Gore, E., Griffin, T.P., Fladeland, M., Bland, G., Soto, C., Madrigal, Y., Castillo, D., Rojas, E., Achi, S.: Utilization of in situ airborne MS-based instrumentation for the study of gaseous emissions at active volcanoes. Int. J. Mass Spectrom. 295, 105-112 (2010)

9. Short, R.T., Fries, D.P., Kerr, M.L.: Influence of the Earth's magnetic field on portable mass spectrometers, in field-portable miniature mass spectrometry. Proceedings of the 12th Sanibel Conference on Mass Spectrometry, Sanibel Island, Florida, 22-25 Jan 2000

10. Teodoro, O., Silva, J., Moutinho, A.: Multitechnique surface analysis system: apparatus description. Vacuum 46, 1205-1209 (1995)

11. Poppenk, F.M., Amini, R., Brouwer, G.F.: Design and application of a Helmholtz cage for testing nano-satellites. Proceedings of the ESA-ESTEC 6th International Symposium Environmental Testing for Space Programme, Noordwijk, The Netherlands, 12-14 June 2007

12. Goldwasser, S.: TV and monitor CRT (Picture Tube) information (v2.02), Samuel M. Goldwasser (1998)

13. Davey, N.G., Krogh, E.T., Gill, C.G.: Membrane-introduction mass spectrometry (MIMS). TrAC Trends Anal. Chem. 30, 1477-1485 (2011)

14. Ketola, R.A., Kotiaho, T., Cisper, M.E., Allen, T.M.: Environmental applications of membrane introduction mass spectrometry. J. Mass Spectrom. 37, 457-476 (2002)

15. Johnson, R.C., Cooks, R.G., Allen, T.M., Cisper, M.E., Hemberger, P.H.: Membrane introduction mass spectrometry: trends and applications. Mass Spectrom. Rev. 19, 1-37 (2000)

16. Short, R.T., Fries, D.P., Toler, S.K., Lembke, C.E., Byrne, R.H.: Development of an underwater mass-spectrometry system for in situ chemical analysis. Meas. Sci. Technol. 10, 1195-1201 (1999)

17. Wenner, P.G., Bell, R.J., van Amerom, F.H.W., Toler, S.K., Edkins, J.E., Hall, M.L., Koehn, K., Short, R.T., Byrne, R.H.: Environmental chemical mapping using an underwater mass spectrometer. TrAC Trends Anal. Chem. 23, 288-295 (2004) 
18. Jenkins, R.: A review of thermionic cathodes. Vacuum 19, 353-359 (1969)

19. Kiser, R.W.: Introduction to mass spectrometry and its applications, (2nd ed.), pp. 195-197. Prentice-Hall, Inc, Upper Saddle River, NJ (1965)
20. Honig, R.E.: Ionization potentials of some hydrocarbon series. J. Chem. Phys. 16, 105-112 (1948)

21. Ørsnes, H., Bohatka, S., Degn, H.: Reaction of water at hot filament interferes with measurements of dissolved gases by membrane inlet mass spectrometry. Rapid Commun. Mass Spectrom. 11, 1736-1738 (1997) 